VoL. $70(2004)$ [199-205]

\title{
DIRECT PRODUCTS AND PROPERLY 3-REALISABLE GROUPS
}

\author{
Manuel Cárdenas, Francisco F. Lasheras and Ranja Roy
}

\begin{abstract}
In this paper, we show that the direct product of infinite finitely presented groups is always properly 3-realisable. We also show that classical hyperbolic groups are properly 3-realisable. We recall that a finitely presented group $G$ is said to be properly 3-realisable if there exists a compact 2-polyhedron $K$ with $\pi_{1}(K) \cong G$ and whose universal cover $\widetilde{K}$ has the proper homotopy type of a (p.l.) 3-manifold with boundary. The question whether or not every finitely presented is properly 3-realisable remains open.
\end{abstract}

\section{INTRODUCTION}

The following question was formulated in [9] for an arbitrary finitely presented group $G$ : does there exist a compact 2-polyhedron $K$ with $\pi_{1}(K) \cong G$ and whose universal cover $\widetilde{K}$ is proper homotopy equivalent to a 3-manifold? If so, the group $G$ is said to be properly 3-realisable. It is known that the proper homotopy type of any locally finite 2-dimensional CW-complex can be represented by a subpolyhedron in $\mathbf{R}^{4}$ (see [4]), thus $\widetilde{K}$ would always be proper homotopy equivalent to a 4-manifold. The question of whether or not every finitely presented $G$ group is properly 3-realisable still remains open. In case of a positive answer, this property would allow us to use duality arguments in the study of certain low-dimensional ((co)homological) proper invariants of the group $G$, see [9]. There are several results in the literature regarding the proper 3-realisability question for finitely presented groups (see $[\mathbf{1}, \mathbf{5}, \mathbf{9}, \mathbf{1 0}]$ ). See also [6] for a survey on this question. In this paper, we prove the following.

THEOREM 1.1. If $G$ and $H$ are infinite finitely presented groups, then the direct product $G \times H$ is properly 3-realisable.

Observe that if $G$ is properly 3-realisable and $H$ is finite, then the direct product $G \times H$ has a copy of $G$ as a subgroup of finite index and hence it is properly 3-realisable, by $([1$, Theorem 1.1]).

COROLlaRY 1.2. Every finitely generated Abelian group is properly 3-realisable.

The techniques used in the Proof of Theorem 1.1 also yield the following.

Received 3rd December, 2003

This work was partially supported by the project BFM 2001-3195-C02.

Copyright Clearance Centre, Inc. Serial-fee code: 0004-9727/04 \$A2.00+0.00. 
PROPOSITION 1.3. If $G$ is the fundamental group of a manifold which can be covered by an Euclidean space, then $G$ is properly 3-realisable.

As an example, we have that all "classical" hyperbolic groups (that is, the fundamental group of a closed Riemannian manifold with negative sectional curvature) are properly 3-realisable.

\section{Preliminaries}

In order to prove Theorem 1.1, we first need some preliminaries from proper homotopy theory. In what follows, we shall be working within the category tow - Gr of towers of groups whose objects are inverse sequences of groups

$$
\underline{A}=\left\{A_{0} \stackrel{\phi_{1}}{\longleftarrow} A_{1} \stackrel{\phi_{2}}{\longleftarrow} A_{2} \longleftarrow \cdots\right\}
$$

A morphism in this category will be called a pro-morphism. See $[\mathbf{2}, \mathbf{1 1}]$ for a general reference.

A tower $L$ is a free tower if it is of the form

$$
\underline{L}=\left\{L_{0} \stackrel{i_{1}}{\longleftarrow} L_{1} \stackrel{i_{2}}{\longleftarrow} L_{2} \longleftarrow \cdots\right\}
$$

where $L_{i}=\left\langle B_{i}\right\rangle$ are free groups of basis $B_{i}$ such that $B_{i+1} \subset B_{i}$, the differences $B_{i}-B_{i+1}$ are finite and $\bigcap_{i=0}^{\infty} B_{i}=\emptyset$, and the bonding homomorphisms $i_{k}$ are given by the corresponding basis inclusions. On the other hand, a tower $\underline{P}$ is a telescopic tower if it is of the form

$$
\underline{P}=\left\{P_{0} \stackrel{p_{1}}{\longleftarrow} P_{1} \stackrel{p_{2}}{\longleftarrow} P_{2} \longleftarrow \cdots\right\}
$$

where $P_{i}=\left\langle D_{i}\right\rangle$ are free groups of basis $D_{i}$ such that $D_{i-1} \subset D_{i}$, the differences We shall also use the full subcategory $(\mathrm{Gr}$, tow $-\mathrm{Gr}$ ) of $\operatorname{Mor}($ tow $-\mathrm{Gr}$ ) whose objects are arrows $\underline{A} \longrightarrow G$, where $\underline{A}$ is an object in tow $-\mathrm{Gr}$ and $G$ is a group regarded as a constant tower whose bonding maps are the identity. Morphisms in ( $\mathrm{Gr}$, tow $-\mathrm{Gr}$ ) will also be called pro-morphisms.

From now on, $X$ will be a (strongly) locally finite CW-complex. A proper map $\omega:[0, \infty) \longrightarrow X$ is called a proper ray in $X$. We say that two proper rays $\omega, \omega^{\prime}$ define the same end if their restrictions $\left.\omega\right|_{\mathbf{N}},\left.\omega^{\prime}\right|_{\mathbf{N}}$ are properly homotopic. Moreover, we say that they define the same strong end if $\omega$ and $\omega^{\prime}$ are in fact properly homotopic.

Given a base ray $\omega$ in $X$ and a collection of compact subsets $C_{1} \subset C_{2} \subset \cdots \subset X$ so that $X=\bigcup_{n=1}^{\infty} C_{n}$, the following tower

$$
\text { pro }-\pi_{1}(X, \omega)=\left\{\pi_{1}(X, \omega(0)) \leftarrow \pi_{1}\left(X-C_{1}, \omega\left(t_{1}\right)\right) \leftarrow \pi_{1}\left(X-C_{2}, \omega\left(t_{2}\right)\right) \leftarrow \cdots\right\}
$$

can be regarded as an object in $(\mathrm{Gr}$, tow $-\mathrm{Gr})$ and it is called the fundamental pro-group of $(X, \omega)$, where $\omega\left(\left[t_{i}, \infty\right)\right) \subset X-C_{i}$ and the bonding homomorphisms are induced by 
the inclusions. This tower does not depend (up to pro-isomorphism) on the sequence of subsets $\left\{C_{i}\right\}_{i}$. It is worth mentioning that if $\omega$ and $\omega^{\prime}$ define the same strong end, then pro $-\pi_{1}(X, \omega)$ and pro $-\pi_{1}\left(X, \omega^{\prime}\right)$ are pro-isomorphic. In particular, we may always assume that $\omega$ is a cellular map. Moreover, if $X$ is strongly connected at each end (that is, any two proper rays defining the same end define the same strong end), then $\pi_{1}^{e}(X, \omega)=\lim _{\leftarrow}$ pro $-\pi_{1}(X, \omega)$ is a well-defined useful invariant which only depends (up to isomorphism) on the end determined by $\omega$ (see [8]). In a similar way, one can define objects in $(\mathrm{Gr}$, tow $-\mathrm{Gr})$ corresponding to the higher homotopy pro-groups of $(X, \omega)$.

Definition 2.1: Given $n \geqslant 1$, a tree $T$ and a proper ray $\omega:[0, \infty) \longrightarrow T$, a spherical object $S_{\omega}^{n}$ under $T$ is a space obtained from $T$ by attaching finitely $n$-spheres $S^{n}$ at each vertex of $\omega([0, \infty))$. Observe that any two of such spherical objects (along $\omega$ ) are proper homotopy equivalent (under $T$ ), by ([2, Propisition 4.5(b)]).

The following result, which characterises those one-ended 2-dimensional proper coH-spaces, will be crucial for the Proof of Theorem 1.1.

THEOREM 2.2. [7, Corollary 6.4]. If $X$ is a one-ended 2-dimensional locally finite $C W$-complex, then the following are equivalent

(a) pro $-\pi_{1}(X, \omega)$ is pro-isomorphic to a (coproduct) tower of the form $\underline{L} \vee \underline{P}$.

(b) There exist spherical objects $S_{\omega}^{2}$ and $S_{\omega^{\prime}}^{2}$ and a proper homotopy equivalence (under $[0, \infty)) X \vee S_{\omega}^{2} \simeq B(\underline{L} \vee \underline{P}) \vee S_{\omega^{\prime}}^{2}$.

Here, $\left(B(\underline{L} \vee \underline{P}), \omega^{\prime}\right)$ is the properly based 2-polyhedron defined as the proper wedge (that is, along a base ray) of a one-ended spherical object $S_{\varepsilon}^{1}$, with pro $-\pi_{1}\left(S_{\varepsilon}^{1}, \omega^{\prime}\right) \cong \underline{L}$ $\left(\omega^{\prime}:[0, \infty) \hookrightarrow S_{\varepsilon}^{1}\right.$ the canonical inclusion), and a proper wedge $C$ of a decreasing sequence (possibly infinite) of cylinders $C_{n}=S^{1} \times[n, \infty)$ and/or Euclidean planes $\mathbf{R}_{m}^{2}=S^{1}$ $\times[m, \infty) / S^{1} \times\{m\}$ attached along the half line $[0, \infty)$ for which pro $-\pi_{1}\left(C, \omega^{\prime}\right) \cong \underline{P}$, with $\omega^{\prime}:[0, \infty) \hookrightarrow C$ the canonical inclusion. Thus, $B(\underline{L} \vee \underline{P})$ can be seen as a "proper Eilenberg-MacLane space" $K(\underline{L} \vee \underline{P}, 1)$.

\section{DIRECT PRODUCTS}

The purpose of this section is to prove Theorem 1.1 and Proposition 1.3. First, we shall roughly outline the generalised van Kampen theorem in a naive way $($ see $[3,12]$ for a proof using groupoids). For simplicity, we shall not take care of base points in what follows (see [14] for details).

Let $X_{0}, X_{1}, X_{2}$ be subcomplexes of a CW-complex $X$ so that $X_{1}, X_{2}$ are connected and satisfy $X=X_{1} \cup X_{2}, X_{1} \cap X_{2}=X_{0}$. Suppose $X_{0}$ is not connected, say it has two connected components $Y$ and $Z$. Let $\tilde{Z}$ denote the CW-complex obtained by identifying a copy of $X_{1}$ with a copy of $X_{2}$ along $Z$, and let $\tilde{X}$ denote the CW-complex (homotopy equivalent to $X$ ) obtained from $Y \times I$ and $\widetilde{Z}$ by identifying $Y \times\{i\}$ to the copy $Y_{i+1}$ of $Y$ in $X_{i+1}, i=0,1$. Then, one can check that we have the following push-out diagrams 
in the category of groups:
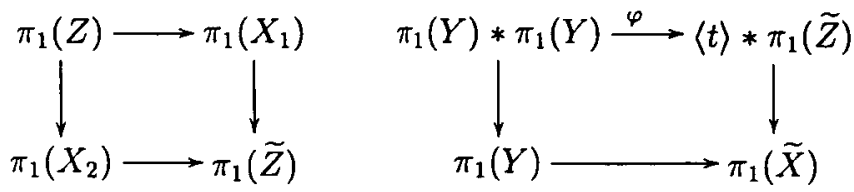

where $t$ is represented by a loop $\left(\left\{y_{0}\right\} \times I\right) \cup \gamma$, with $y_{0} \in Y$ and $\gamma$ a path in $\tilde{Z}$ from $\left(y_{0}, 0\right)$ to $\left(y_{0}, 1\right)$; and $\varphi$ is given by $\theta \mapsto \theta$ on the first factor, and by $\theta \mapsto t \theta t^{-1}$ on the second factor. From now on, we shall denote $\pi_{1}\left(X_{1}\right) \widehat{*}_{\pi_{1}(Z)} \pi_{1}\left(X_{2}\right) \equiv \pi_{1}(\widetilde{Z})$ and $\pi_{1}(\widetilde{Z}) \widehat{*}_{\pi_{1}(Y)}$ $\equiv \pi_{1}(\tilde{X}) \cong \pi_{1}(X)$ the corresponding fundamental groups obtained by the process described above.

Proposition 3.1. Let $X$ and $Y$ be locally finite, simply connected noncompact $C W$-complexes. Then, pro $-\pi_{1}(X \times Y)$ is pro-isomorphic to a telescopic tower $\underline{P}$.

Corollary 3.2. With $X$ and $Y$ as above, we have $\lim ^{i}$ pro $-\pi_{1}(X \times Y)=\{1\}$.

Note that Mihalik [13] already showed that the product $X \times Y$ of locally finite, connected non-compact $\mathrm{CW}$-complexes is semistable at $\infty$.

ProOf of Proposition 3.1: Let $X$ and $Y$ be locally finite, simply connected noncompact CW-complexes. In [8], the computation of pro $-\pi_{1}(X \times Y)$ is done in detail for $Y=\mathbf{R}$ (and $X$ not necessarily simply connected). The computations in the general case we are concerned with are similar to those in [8], so we shall not take care of base rays or base points in what follows, for simplicity. Notice that $X \times Y$ is strongly connected at infinity, that is, it only has one strong end (see [13]).

Let $C_{1} \subset C_{2} \subset \cdots \subset X$ and $D_{1} \subset D_{2} \subset \cdots \subset Y$ be sequences of compact subsets with $X=\bigcup_{i=1}^{\infty} C_{i}$ and $Y=\bigcup_{i=1}^{\infty} D_{i}$, and so that $X-C_{i}=C_{i, 1} \cup \cdots \cup C_{i, m_{i}}$ and $Y-D_{i}=D_{i, 1} \cup \cdots \cup D_{i, n_{i}}$ are the corresponding collections of connected components satisfying $C_{i+1,1} \subset C_{i, 1}$, for all $i$, and $C_{i+1,2} \subset C_{i, 2}$ if $m_{i} \geqslant 2$.

Consider $U_{i}=(X \times Y)-\left(C_{i} \times D_{i}\right)=\left(\bigcup_{j=1}^{n_{i}} X \times D_{i, j}\right) \cup\left(\bigcup_{j=1}^{m_{i}} C_{i, j} \times Y\right), i \geqslant 1$. We wish to compute $\pi_{1}\left(U_{i}\right)$ as well as the bonding homomorphism $\pi_{1}\left(U_{i+1}\right) \longrightarrow \pi_{1}\left(U_{i}\right)$ induced by inclusion. By the generalised van Kampen theorem, $\pi_{1}\left(U_{i}\right)$ can be expressed as follows (the picture roughly describes the case $m_{i}=n_{i}=2$ ):

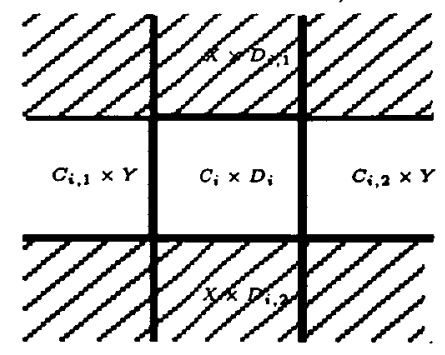




$$
\begin{array}{r}
\left(\left\{\left(\pi_{1}\left(X \times D_{i, 1}\right) \widehat{*}_{\pi_{1}}\left(C_{i, 1} \times D_{i, 1}\right) \pi_{1}\left(C_{i, 1} \times Y\right)\right) \cdots \widehat{*}_{\pi_{1}\left(C_{i, m_{i}} \times D_{i, 1}\right)} \pi_{1}\left(C_{i, m_{i}} \times Y\right)\right.\right. \\
\left.\left.\widehat{*}_{\pi_{1}\left(C_{i, 1} \times D_{i, 2}\right)} \pi_{1}\left(X \times D_{i, 2}\right) \cdots \widehat{*}_{\pi_{1}\left(C_{i, 1} \times D_{i, n_{i}}\right)} \pi_{1}\left(X \times D_{i, n_{i}}\right)\right\} \widehat{*}_{\pi_{1}\left(C_{i, 2} \times D_{i, 2}\right)}\right) \\
\cdots \widehat{*}_{\pi_{1}\left(C_{i, 2} \times D_{i, n_{i}}\right) \ldots} \widehat{*}_{\pi_{1}\left(C_{i, m_{i}} \times D_{i, 2}\right)} \cdots \widehat{*}_{\pi_{1}\left(C_{i, m_{i}} \times D_{i, n_{i}}\right)}
\end{array}
$$

Moreover, if we take $P_{i}=F\left(\left\{t_{i, j, k}, 2 \leqslant j \leqslant m_{i}, 2 \leqslant k \leqslant n_{i}\right\}\right)$ (here, $F(A)$ stands for the free group on the set $A$ ), then there are homomorphisms $\alpha_{i}: P_{i} \longrightarrow \pi_{1}\left(U_{i}\right)$ and commutative diagrams

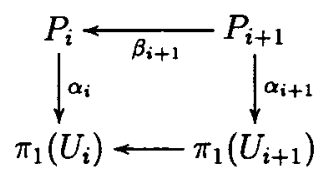

where:

(i) $\alpha_{i}$ maps each $t_{i, j, k}$ to the new generator added when considering the corresponding push-out $(. ..) \widehat{*}_{\pi_{1}\left(C_{i, j} \times D_{i, k}\right)}$ in the expression of $\pi_{1}\left(U_{i}\right)$ given above.

(ii) $\beta_{i+1}\left(t_{i+1, j, k}\right)=t_{i, j^{\prime}, k^{\prime}}$ whenever $C_{i+1, j} \subset C_{i, j^{\prime}}\left(j^{\prime} \geqslant 2\right)$ and $D_{i+1, k} \subset D_{i, k^{\prime}}$ $\left(k^{\prime} \geqslant 2\right)$.

(iii) $\beta_{i+1}\left(t_{i+1, j, k}\right)=1$ if $C_{i+1, j} \subset C_{i, 1}$.

Observe that the group inside the curly brackets in the expression of $\pi_{1}\left(U_{i}\right)$ given above is the trivial group, since $X$ and $Y$ are both simply connected and hence the group homomorphisms involved in the corresponding push-out diagrams can be regarded as induced by the corresponding projections $C_{i, j} \times D_{i, k} \longrightarrow C_{i, j}$ and $C_{i, j} \times D_{i, k} \longrightarrow D_{i, k}$. Moreover, it is not hard to check that each homomorphism $\alpha_{i}: P_{i} \longrightarrow \pi_{1}\left(U_{i}\right)$ is in fact an isomorphism, with the above considerations.

Finally, after an appropriate change of basis for the free groups $P_{i}$ (in terms of the original generators $t_{i, j, k}$ ), one can see that the tower

$$
\underline{P}=\left\{\{1\} \longleftarrow P_{1} \stackrel{\beta_{2}}{\longleftarrow} P_{2} \stackrel{\beta_{3}}{\longleftarrow} P_{3} \longleftarrow \cdots\right\}
$$

can be regarded as a telescopic tower, and the conclusion of the proposition follows.

ProOF OF THEOREM 1.1: Let $G$ and $H$ be infinite finitely presented groups, and let $X$ and $Y$ be compact 2-polyhedra with $\pi_{1}(X) \cong G$ and $\pi_{1}(Y) \cong H$. Let $\tilde{X}$ and $\tilde{Y}$ denote the universal covers of $X$ and $Y$ respectively. Observe that $\tilde{X}$ and $\tilde{Y}$ are noncompact polyhedra, since $G$ and $H$ are infinite. It is clear that $\pi_{1}(X \times Y) \cong G \times H$ and $\tilde{X} \times \tilde{Y}$ is the universal cover of $X \times Y$. Let $p: \widetilde{X} \times \tilde{Y} \longrightarrow X \times Y$ be the universal covering projection and let $W$ denote the 2-skeleton of $X \times Y$. Then, $\pi_{1}(W) \cong G \times H$ and $\widetilde{W}=p^{-1}(W) \subset \widetilde{X} \times \widetilde{Y}$ is the universal cover of $W$, with pro $-\pi_{1}(\widetilde{W}) \equiv$ pro $-\pi_{1}(\widetilde{X} \times \tilde{Y})$ which is pro-isomorphic to a telescopic tower $\underline{P}$, by Proposition 3.1. Pick a base ray $\omega$ in 
$\widetilde{W}$. Since $\widetilde{W}$ is 2-dimensional and (strongly) one-ended, there exist spherical objects $S_{\omega}^{2}$ and $S_{\omega^{\prime}}^{2}$ and a proper homotopy equivalence $\widetilde{W} \vee S_{\omega}^{2} \simeq B(\underline{P}) \vee S_{\omega^{\prime}}^{2}$, by Theorem 2.2 . Let $V \subset \widetilde{W}$ be the set of vertices in $\omega([0, \infty))$, with $p(V)=\left\{v_{1}, \ldots, v_{r}\right\} \subset W$, and denote by $\widehat{W}$ the polyhedron obtained from $\widetilde{W} \vee S_{\omega}^{2}$ by attaching one sphere $S^{2}$ through every vertex in $p^{-1}(p(V))-\omega([0, \infty))$. Thus, $\widehat{W}$ is the universal cover of the compact 2-polyhedron obtained from $W$ by attaching one sphere $S^{2}$ at each of the vertices $v_{1}, \ldots, v_{r}$ (which is homotopy equivalent to a wedge $W \vee\left(\bigvee_{i=1}^{r} S^{2}\right)$ ). On the other hand, $\widehat{W}$ is proper homotopy equivalent to a polyhedron $Q$ obtained from $B(\underline{P}) \vee S_{\omega^{\prime}}^{2}$ by attaching infinitely many spheres $S^{2}$ in a proper way (that is, via the corresponding proper homotopy equivalence given by Theorem 2.2 ). Finally, the proper homotopy type of the proper wedge $B(\underline{P}) \vee S_{\omega^{\prime}}^{2}$ can be represented by the closed subpolyhedron in $\mathbf{R}^{3}$ shown in the figure below. It is then easy to check that the proper homotopy type of $Q$ can also be represented by a closed subpolyhedron $\widehat{Q}$ in $\mathbf{R}^{3}$.

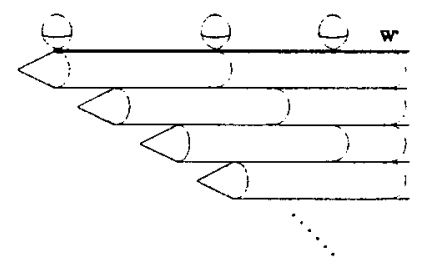

Therefore, the universal cover of the compact 2-polyhedron $W \vee\left(\bigvee_{i=1}^{r} S^{2}\right)$ (with $\pi_{1}(W$ $\left.\left.\vee\left(\bigvee_{i=1}^{r} S^{2}\right)\right) \cong G \times H\right)$ turns out to be proper homotopy equivalent to the 3-manifold obtained by taking a regular neighbourhood of $\widehat{Q}$ in $\mathbf{R}^{3}$, and the conclusion of the theorem follows.

Proof of Proposition 1.3: Let $G$ be the fundamental group of an $n$-manifold $M$ whose universal cover $\widetilde{M}$ can be identified with the Euclidean space $\mathbf{R}^{n}$. Thus, pro $-\pi_{1}(\widetilde{M})$ is clearly pro-isomorphic to a telescopic tower. Therefore, using an argument similar to that of Theorem 1.1, there is a finite wedge $\bigvee_{i \in I} S^{2}$ so that the universal cover of $W \vee\left(\bigvee_{i \in I} S^{2}\right)$ is proper homotopy equivalent to a 3-manifold, where $W$ is the 2-skeleton of $M$.

\section{REFERENCES}

[1] R. Ayala, M. Cárdenas, F.F. Lasheras and A. Quintero, 'Properly 3-realizable groups', Proc. Amer. Math. Soc. (to appear).

[2] H-J. Baues and A. Quintero, Infinite homotopy theory, K-monographs in Mathematics 6 (Kluwer Academic Publishers, Dordrecht, 2001). 
[3] R. Brown, Elements of modern topology (McGraw-Hill, New York, 1968).

[4] M. Cárdenas, T. Fernández, F. F. Lasheras and A. Quintero, 'Embedding proper homotopy types', Colloq. Math. 95 (2003), 1-20.

[5] M. Cárdenas and F.F. Lasheras, 'On properly 3-realizable groups', Topology Appl. (to appear).

[6] M. Cárdenas and F.F. Lasheras, 'Properly 3-realizable groups : a survey', in Proceedings of the Conference on Geometric Group Theory and Geometric Methods in Group Theory (Seville 2003), Contemp. Math. (to appear).

(7) M. Cárdenas, F.F. Lasheras, F. Muro and A. Quintero, 'Proper L-S category, fundamental pro-groups and 2-dimensional proper co- $H$-spaces', (preprint).

[8] R. Geoghegan and M. Mihalik, 'The fundamental group at infinity', Topology 35 (1996), 655-669.

[9] F.F. Lasheras, 'Universal covers and 3-manifolds', J. Pure Appl. Algebra 151 (2000), 163-172.

[10] F. F. Lasheras, 'A note on fake surfaces and universal covers', Topology Appl. . 125 (2002), 497-504.

[11] S. Mardešic and J. Segal, Shape theory, North-Holland Mathematical Library 26 (North-Holland, Amsterdam, New York 1982).

[12] P.J. May, $A$ concise course in algebraic topology, Chicago Lectures in Mathematics (University of Chicago Press, Chicago, IL, 1999).

[13] M. Mihalik, 'Semistability at the end of a group extension', Trans. Amer. Math. Soc. 277 (1983), 307-321.

[14] P. Scott and C.T.C. Wall, 'Topological methods in group theory', in Homological Group Theory, London Math. Soc. Lecture Notes (Cambridge Univ. Press 36, Cambridge, New York, 1979), pp. 137-204.

Departamento de Geometría y Topologia
Universidad de Sevilla, Apdo 1160
41080-Sevilla
Spain
e-mail: lasheras@us.es, mcard@us.es
New York Institute of Technology

Old Westbury, NY 11568-8000

United States of America

e-mail: rroy@nyit.edu 